\title{
Determination of iodine value of palm oil based on triglyceride composition
}

\begin{abstract}
The triglyceride (TG) composition of palm oil is normally determined by high-performance liquid chromatography (HPLC). The HPLC chromatograms indicated a good separation of most of the TG components in the oil. The TG can be classified based on either the TG groups, i.e., triunsaturated, monosaturated, disaturated, or trisaturated, or the number of double bonds, i.e., zero, one, two, three, or four double bonds. The more unsaturated the fatty acid, the greater the iodine value (IV). Therefore, it is hypothesized that the IV of an oil can be determined based upon the TG composition of the oil. Based on the TG groups, stepwise regression analysis showed that the areas of the disaturated, trisaturated, and triunsaturated TG peaks could predict the IV with a coefficient of determination (R2) of 0.990 . The regression based on the number of double bonds yielded a good regression equation with $\mathrm{R} 2=0.992$. The important variables were the peak area of the fatty acids that contained zero, one, two, and three double bonds. This study concludes that the TG composition can be used to predict the IV of palm oil. The best prediction model is obtained by using the number of double bonds in the TG as the independent variable.
\end{abstract}

Keyword: HPLC; Instrumental analysis; Iodine value; Palm oil; Triglyceride 